\section{El embarazo no deseado: impacto sobre la salud y la sociedad en América Latina y el Caribe}

\author{
Ana Langer ${ }^{1}$
}

Palabras clave: embarazo no deseado, aborto inseguro, mortalidad materna, anticonceptivos, planificación familiar, América Latina y Caribe.

\footnotetext{
1 Population Council, Oficina Regional para América Latina y el Caribe. Escondida \# 110, Col. Villa Coyoacán, México, D.F. 04000, México.
}

\section{EL EMBARAZO NO DESEADO: CONCEPTOS Y SITUACIÓN REGIONAL}

La reproducción y su condición necesaria, el ejercicio de la sexualidad, deberían ser siempre actos deseados y planeados. Lamentablemente, no es así. Prueba de ello son los embarazos no deseados, definidos como aquellos que ocurren en un momento poco favorable, inoportuno, o que se dan en una persona que ya no quiere reproducirse. ¿Por qué, en una época en la que, al menos en teoría, existen los medios para regular la fecundidad, las mujeres aún siguen teniendo este problema?

- Porque las mujeres y las parejas de América Latina y el Caribe (ALC) desean menos hijos que los que naturalmente resultan del ejercicio de una vida sexual activa. Las encuestas de demografía y salud que se han llevado a cabo en la mayoría de los países de la Región en las últimas décadas muestran que, en promedio, el tamaño de la familia se ha reducido de manera importante en los últimos 30 años: de 6 hijos en 1960-1965 a 3,3 en el primer quinquenio de los años noventa (1). Sin embargo, el número de hijos que las mujeres desearían tener es todavía menor. Así, por ejemplo, en Bolivia, en 1998 el tamaño medio de la descendencia era de 4,2 hijos, mientras que el número medio de hijos que las mujeres mencionaban como ideal era de 2,6 (2). En Colombia, en el año 2000, el tamaño de la familia era en promedio de 2,6 hijos, mientras que el número deseado de hijos era, en promedio, de 2,3 (3). Estos datos, de por sí ilustrativos, son medias nacionales que ocultan grandes diferencias según la edad, escolaridad y lugar de residencia. Por ejemplo, en México, en 1997 las mujeres de 15 a 19 años declararon que su número ideal de hijos era, en promedio, de 2,7, mientras que las del grupo de 45 a 49 años respondieron que era de 4; aquellas sin escolaridad dijeron que el número ideal era de 4,3 y las que tenían educación secundaria o superior aspiraban a 2,7. Finalmente, las de origen rural señalaron que su tamaño ideal de descendencia era de 3,8 y las de origen urbano, de 3 (4). En todos los países donde se ha recogido este tipo de información se han observado tendencias similares.

Este cambio refleja profundas y complejas transformaciones sociales y culturales a las que con- 
tribuyen un sinnúmero de circunstancias, entre ellas el descenso de la mortalidad infantil y mayores expectativas de los padres para el desarrollo personal de los hijos, las crecientes escolaridad y participación de la mujer en el mercado laboral, las políticas de población y los mensajes oficiales sobre el valor de una familia menos numerosa, así como las características de la vida urbana.

Las expectativas reproductivas están influenciadas por la cultura y la ideología, y difieren según el grupo social y el contexto histórico. El papel que se les asigna a las mujeres en la sociedad está íntimamente relacionado con las expectativas reproductivas y varía dentro de una amplia gama que va desde el papel exclusivo de madre y cuidadora de los hijos hasta su desempeño pleno como trabajadora o profesional.

- Porque aún no todas las personas pueden controlar su fecundidad. En la segunda mitad del siglo XX aparecieron y se desarrollaron los métodos anticonceptivos modernos, que son muy eficaces y seguros y que, por primera vez en la historia de la humanidad, permitieron llevar las expectativas reproductivas a la práctica. En efecto, la anticoncepción moderna es la herramienta idónea para que las mujeres y las parejas puedan elegir el momento en que inician la reproducción, el número de hijos que van a tener y el espaciamiento entre los embarazos.

En la Región se ha observado una tendencia constante al aumento del uso de métodos anticonceptivos. Así, por ejemplo, en Bolivia (uno de los países con una prevalencia de uso más baja) la proporción de mujeres en edad fértil que utiliza anticoncepción ha aumentado del 30,3\% en 1989 (5) al $45,3 \%$ en 1994 (6) y al 48,3\% en 1998 (2), y en Nicaragua del 49\% en 1993 (7) al 60,3\% en 1998 (8). En el otro extremo del espectro (países con frecuencias de uso elevadas), Colombia muestra un aumento del $72 \%$ en 1995 (9) al 77\% en 2000 (3) y en México se observa una tendencia ascendente sin interrupciones: del 63,1\% en 1992 al 66,5\% en 1996 y al 70,8\% en 2000 (4).

Sin embargo, en la práctica, existen mujeres que no desean reproducirse pero siguen expuestas al "riesgo" de quedar embarazadas, por estar en edad reproductiva y llevar una vida sexual activa sin usar ningún método anticonceptivo o porque utilizan métodos "tradicionales" como el ritmo o el coito interrupto, de escasa eficacia para evitar el embarazo. Demógrafos y especialistas en reproducción se refieren a este grupo de la población como aquellas personas con una "necesidad no satisfecha de planificación familiar o anticoncepción". La pro- porción de mujeres que se encuentra en esta situación no es despreciable: en Bolivia, por ejemplo, ascendía al 26\% en 1998 (2), en Nicaragua al 14,7\% en 1998 (8) y en México al 12,1\% en 1997 (10). Según el Instituto Alan Guttmacher, en 1994 existían 8 millones de mujeres con esta necesidad insatisfecha en Brasil, 1,8 millones en Colombia, 6,7 millones en México, 1,9 millones en Perú y 351000 en la República Dominicana (1).

Diversas encuestas realizadas en distintos países proporcionan algunas indicaciones sobre las razones que subyacen a esta situación (1). La falta de conocimientos sobre los anticonceptivos es una de ellas, si bien en los últimos años se menciona menos frecuentemente que antes. En cambio, contribuye más el conocimiento deficiente de cómo utilizar un método determinado (especialmente los anticonceptivos orales), resultado de la ausencia total de asesoramiento (esto sucede, por ejemplo, cuando se adquieren los anticonceptivos en la farmacia y no se accede a una consulta con una persona cualificada) o de un asesoramiento deficiente. Este desconocimiento hace que el fracaso de los métodos sea frecuente.

Otras razones que explican la "necesidad no satisfecha" son el temor (infundado, por cierto) a los efectos secundarios de los métodos, la oposición de la pareja, la postura de algunas iglesias (sobre todo la Católica y grupos asociados con ella) y las barreras para conseguir los métodos (inexistencia de servicios, costos, diferencias culturales o lingüísticas). Estas barreras, junto con la deficiente calidad de los servicios, representan factores particularmente importantes para ciertos grupos de mujeres (rurales e indígenas, sobre todo), los hombres y los adolescentes, cuyas necesidades de atención son diferentes de las de la mayoría de la población usuaria.

- Porque existen relaciones sexuales que no son voluntarias ni deseadas, cuyas expresiones extremas son la violación y la violencia sexual, o cuando existe una fuerte presión social para el inicio de la vida sexual, como sucede en algunos grupos de adolescentes. Mientras existan relaciones no planeadas y sin el consentimiento de la mujer, existirán los embarazos no deseados. Muchos países de la Región permiten la interrupción legal del embarazo en caso de violación, pero aun en estas circunstancias, muchas mujeres se ven obligadas a continuar con un embarazo no deseado secundario a violación debido a que desconocen la ley o a que la organización de los servicios de salud no facilita el acceso a este procedimiento.

- Porque los métodos anticonceptivos fallan y no hay ninguno que sea eficaz en un $100 \%$. Se estima que cada año ocurren en el mundo entre 8 y 30 millo- 
nes de embarazos debidos al fracaso de los métodos anticonceptivos o a su uso incorrecto (11). Si bien una mejor información contribuiría a reducir estas cifras, nunca lograrán evitarse totalmente los embarazos no deseados debidos a esta causa.

Los embarazos no deseados que resultan de relaciones sexuales no consensuadas o del fracaso de los métodos anticonceptivos son más de los que deberían, considerando que existe un método seguro y eficaz para prevenir el embarazo tras una relación sexual sin protección: la anticoncepción de emergencia. ${ }^{2}$ Lamentablemente, este recurso no está disponible en toda la Región, debido a que los responsables de políticas y programas de muchos países no lo han aprobado oficialmente por confundir este método anticonceptivo con un método abortivo.

Los embarazos no deseados son especialmente comunes en adolescentes, mujeres solteras y mayores de 40 años; sin embargo, también se dan en otros grupos de edad. Su frecuencia parece ser mayor entre las mujeres de escasos recursos y bajo nivel educativo, aunque ocurren en todas las clases sociales. Sus consecuencias no solo afectan a las mujeres, sino también a los hombres y a las familias.

\section{CONSECUENCIAS DEL EMBARAZO NO DESEADO}

Ante un embarazo no deseado, las mujeres y las parejas enfrentan decisiones difíciles. Las opciones que se abren son básicamente dos:

\footnotetext{
2 La anticoncepción de emergencia (ACE) es el único método poscoital disponible hasta ahora que las mujeres pueden utilizar para prevenir el embarazo después de una relación sexual sin protección, de un accidente anticonceptivo o de una violación. Según demuestran los estudios sobre el tema, su eficacia es del $75 \%$; en otras palabras, la ACE previene 3 de cada 4 embarazos que ocurrirían después de una relación sexual sin protección). La ACE consiste en la ingestión de una dosis especial, más alta, de los anticonceptivos orales que se encuentran en el mercado, en las primeras 72 horas después del coito, y una segunda dosis 12 horas después. En varios países de la región ya existe un producto destinado a la ACE y el método está incluido en las normas oficiales de planificación familiar. Sin embargo, hay otros países donde este método no ha sido aprobado, a pesar de la existencia de políticas de población y salud reproductiva avanzadas, como en el caso de México.

La ACE actúa interrumpiendo el ciclo reproductivo de la mujer y es un método eficaz y seguro, sin efectos secundarios importantes; no afecta al embrión si la mujer que la ingiere ya estaba embarazada, y no es abortiva ni tampoco se recomienda como anticonceptivo de rutina. En cambio, representa una segunda oportunidad para reducir en una gran proporción los embarazos no deseados y sus terribles consecuencias. Es importante que se difunda ampliamente información sobre este método entre las jóvenes y las víctimas de relaciones sexuales no consensuadas, y que las usuarias y usuarios de planificación familiar que se enfrenten a problemas en el uso de los anticonceptivos, como el olvido de la toma de un anticonceptivo hormonal oral o la ruptura o deslizamiento del condón, conozcan esta alternativa.
}

- Intentar interrumpir el embarazo a través de un aborto inducido, con todos los riesgos y consecuencias que este procedimiento conlleva en sociedades en las que está legalmente restringido.

- Continuar con el embarazo no deseado.

Cualquiera de estas decisiones tiene consecuencias sobre la salud y la situación social y económica de la mujer, su pareja y su familia. Además, también influyen en las condiciones de salud y de desarrollo de las sociedades.

Las consecuencias sobre la salud de las mujeres son indirectas: las más graves se derivan del aborto inducido al que las mujeres recurren para tratar de evitar el embarazo no deseado, y que se realiza en condiciones de clandestinidad e inseguridad. La continuación de los embarazos no deseados también tiene consecuencias sobre la salud de la madre y del hijo, ya que estos ocurren con mayor frecuencia en mujeres en ambos extremos de la edad reproductiva, períodos durante los cuales los riesgos son mayores. Los dos tipos de implicaciones sobre la salud se detallan en las secciones que siguen. Finalmente, los embarazos no deseados tienen consecuencias que van más allá del individuo y afectan a toda la sociedad. Estas situaciones también se describen en este trabajo.

\section{Consecuencias del embarazo no deseado sobre la salud}

Aborto inducido. Las consecuencias más graves del embarazo no deseado sobre la salud son los problemas que resultan de un aborto inseguro. En los Estados Unidos de América (EE.UU.) se sabe que aproximadamente la mitad de los embarazos no deseados terminan en aborto (12). Algunas estimaciones señalan que en ALC el aborto inducido es el camino elegido en un 17 a $35 \%$ de los casos (1).

La interrupción voluntaria del embarazo ha ocurrido en todas las culturas y en todas las épocas, a veces legal y culturalmente aceptado, otras rechazado con violencia. Es un problema complejo que puede abordarse desde la ética y la filosofía, el derecho, la política, la sicología, la sociología, la historia y las ciencias de la salud. Sin embargo, cuando se estudia el aborto inducido desde esta última perspectiva, es imprescindible dejar de lado los profundos conflictos que el tema despierta en individuos, parejas y familias y, en general, en la sociedad, para encararlo como uno de los más importantes problemas de salud pública que enfrentamos en la Región. Esta afirmación se sustenta en varias consideraciones que se detallan a continuación. 
El aborto inducido es una de las principales causas de enfermedad y muerte para las mujeres de América Latina y el Caribe. El aborto que se realiza en condiciones inseguras se acompaña con frecuencia de complicaciones secundarias al procedimiento utilizado. Por ejemplo, cuando se introducen objetos no estériles o punzantes en el útero, los riesgos que enfrenta la mujer son enormes, y se agravan cuando esta no cuenta con información que le permita identificar los signos de las complicaciones, cuando retrasa la búsqueda de atención por temor, vergüenza o desconocimiento de adónde acudir, o cuando la calidad de la atención es deficiente. En un estudio realizado en Brasil se estimó que el 20\% de los abortos clandestinos realizados por médicos en clínicas y el $50 \%$ de los efectuados en casas particulares por la propia mujer o por personal sin entrenamiento se complican. (13) Estos datos contrastan con los provenientes de países donde el aborto es legal y se realiza en buenas condiciones, donde solo el 5\% de las mujeres que interrumpen su embarazo sufre algún problema.

Las complicaciones inmediatas más frecuentes del aborto inseguro son la perforación del útero, la hemorragia y la infección. A medio plazo, la infertilidad es una secuela frecuente. Lamentablemente, no se cuenta con datos sistemáticos y precisos para cuantificar la morbilidad que ocurre como consecuencia del aborto inducido, excepto en los casos en los que la mujer acude a un centro hospitalario. Un estudio realizado en el Instituto Mexicano del Seguro Social muestra que el $63 \%$ de los internamientos de pacientes con problemas relacionados con el embarazo corresponden a complicaciones secundarias a un aborto (14). Esta proporción es altísima y está muy por encima de la causa de internamiento que le sigue en frecuencia, la hemorragia (19\%). Todas las mujeres que, bien o mal, resuelven su problema en el hogar o en la comunidad (fuera de los hospitales) no quedan registradas en ninguna estadística oficial.

Cuando se acompaña de sentimientos de culpa y se lleva a cabo en condiciones traumáticas, el aborto inducido también tiene consecuencias psicológicas como depresión, ansiedad y miedo. No obstante, es importante señalar que la continuación de un embarazo no deseado y la experiencia de ser madre contra voluntad también suele tener consecuencias emocionales graves (15). Por ahora no existen cifras en nuestra Región que permitan estimar la frecuencia de las complicaciones emocionales del aborto inducido. No obstante, es posible afirmar que la decisión entre continuar un embarazo no deseado o interrumpirlo es siempre difícil y dolorosa para la mujer y quienes la rodean.

El aborto inducido es también una importante causa de muerte materna (defunciones que ocurren como consecuencia de problemas del embarazo y el parto o de complicaciones surgidas durante el puerperio, o que se derivan de una mala atención). En efecto, cuando un aborto se realiza en condiciones inseguras y la mujer con complicaciones no tiene acceso a atención oportuna y de buena calidad, el riesgo de morir es muy alto.

Conocer el número de muertes que ocurren en América Latina como consecuencia del aborto sería un dato importante para analizar y abordar el problema. Lamentablemente, solo contamos con aproximaciones a la cifra real, debido a la calidad deficiente de nuestras estadísticas oficiales relativas a la mortalidad materna, así como a la clandestinidad del procedimiento.

Actualmente, se está realizando en México un proyecto de investigación que aportará información empírica sobre esta cuestión. ${ }^{3}$ En este proyecto se van a visitar familiares o vecinos de mujeres fallecidas por causas maternas confirmadas o "probablemente maternas" en dos áreas del país; el objetivo es conocer las circunstancias que rodearon la muerte e identificar aquellos casos en los que podrían haber participado las complicaciones de un aborto inducido. Para estas entrevistas se utilizará la técnica de la "autopsia verbal". Dado que el tema es sumamente delicado, las entrevistas se realizarán por personal entrenado específicamente para este propósito y se garantizará la confidencialidad de los informantes. Los datos que se obtengan sobre la contribución del aborto inducido a las muertes maternas permitirán ajustar las cifras nacionales y conocer la verdadera proporción de la mortalidad materna que se debe a esta causa.

En nuestros países la calidad de la información sobre la mortalidad materna es en general deficiente, debido a que una importante proporción de la población aún no accede a instituciones de salud y, en consecuencia, sus muertes no quedan asentadas en ningún registro oficial. Otro factor que contribuye a la mala calidad de la información es la inadecuada clasificación de las muertes maternas como resultado de la deficiente cumplimentación de los certificados de defunción. Esto sucede porque a menudo dichos certificados son rellenados por personas sin entrenamiento o porque los médicos prefieren no incluir datos sobre complicaciones del aborto que podrían atraer nuevas averiguaciones e, incluso, enfrentarlos a consecuencias legales. Además, las muertes maternas que ocurren más de 42 días después del aborto o el nacimiento (aproximadamente el $11 \%$ del total $(16,17)$ generalmente no se incluyen en la categoría de muerte materna.

\footnotetext{
3 Este es un proyecto en marcha que está siendo desarrollado por el Population Council y el Instituto Nacional de Salud Pública de México.
} 
Todas estas circunstancias explican que el 30 a $50 \%$ de las muertes maternas no queden registradas como tal $(18,19)$. En otras palabras, el número real de muertes maternas es aproximadamente del doble de lo que reflejan las estadísticas oficiales.

Es razonable suponer que el subregistro sea aún mucho más importante para las muertes que ocurren como consecuencia de un aborto inducido en condiciones de clandestinidad. En efecto, el temor a la censura y a las consecuencias legales que sienten las mujeres, sus familiares y los profesionales de la salud determina que muchas de estas defunciones queden registradas bajo otras causas, como infección o hemorragia. Estudios realizados en Brasil (20) y Colombia (17) estimaron que alrededor del $60 \%$ de las muertes maternas clasificadas bajo la categoría de "infección" estaban asociadas con un aborto. Otro factor que contribuye al subregistro es que, si el embarazo fue muy reciente, la condición de gravidez ni siquiera se menciona en el certificado.

La mala calidad de la información disponible obliga a recurrir a cálculos indirectos para estimar el número de muertes maternas que ocurren en América Latina como consecuencia del aborto (21). Royston et al. (22) hicieron un ejercicio de este tipo. En primer lugar, tomaron el número de muertes maternas registradas oficialmente como tales y corrigieron estas cifras por el subregistro de la mortalidad materna en general, calculado en un $50 \%$. En pocas palabras, multiplicaron por dos el número de muertes maternas registradas oficialmente. En 1990, año que tomaron los autores como ejemplo, hubo en la Región 14000 muertes maternas que, una vez ajustadas por el subregistro del $50 \%$, ascendieron a 28000 . Según cifras oficiales, aproximadamente el $17 \%$ de la mortalidad materna se debe al aborto inducido, lo que nos daría un número de 4760 (17\% de 28000$)$ mujeres fallecidas a consecuencia de la interrupción del embarazo. Esta cifra todavía está subestimada, ya que falta corregirla por el subregistro de las muertes maternas debidas específicamente al aborto y mal clasificadas bajo los rubros de hemorragia o infección, que se estima en un $50 \%$. Al hacer esta corrección se llega a una cifra de alrededor de $10000(4760 \times 2)$ muertes por aborto por año en América Latina.

El lugar que ocupa el aborto entre las causas de muerte materna varía en los distintos países, con proporciones más elevadas en el Cono Sur. Es evidente que las muertes por aborto son menos frecuentes cuando el uso de anticonceptivos es más alto (y, por lo tanto, es menor la proporción de embarazos no deseados), y cuando la calidad de los servicios para la atención de las complicaciones es mejor (23). Tomando en cuenta esta variación, es posible estimar que ocurren entre 83 y 250 muertes por cada 100000 abortos. En promedio, en los países donde el aborto es legal, la cifra es de 0,6 por cada 100000 interrupciones del embarazo (21).

Las defunciones a consecuencia de un aborto mal practicado son muertes de mujeres jóvenes, responsables del cuidado de hijos pequeños y de otros miembros de la familia. Además son muertes totalmente prevenibles, ya que se podría evitar el embarazo no deseado, contar con procedimientos seguros para la interrupción del mismo y tratar con eficacia las complicaciones del aborto, lo que hace que esta realidad sea verdaderamente inaceptable.

La atención a las complicaciones del aborto consume importantes recursos del sector de la salud. De hecho, la atención de estos problemas ocupa el segundo lugar en uso de recursos hospitalarios para la atención obstétrica, después del parto normal, que es el motivo más común por el que las mujeres en edad reproductiva acuden a una institución de salud. El costo de la atención a las complicaciones del aborto en distintas instituciones y circunstancias es muy variable y no se ha estimado de una manera sistemática. Para aproximarse a una cifra sería necesario considerar los costos hospitalarios directos que resultan de la atención de complicaciones graves, más las implicaciones financieras de un aborto complicado para la mujer, su familia y la comunidad. En algunos contextos, el costo de un aborto clandestino realizado por un médico en una clínica equivale a los ingresos anuales de una familia media. Evidentemente, para un análisis cuidadoso de los costos habría que asignar un valor monetario a la muerte materna y a las consecuencias de la desaparición de la mujer sobre la familia, la vida de sus hijos y la comunidad. Atribuir costos a estas situaciones es un asunto complejo que los economistas de la salud aún no han resuelto.

Más allá de la variabilidad que seguramente existe entre países y grupos sociales, se puede afirmar sin temor a equivocarse que los costos derivados de incrementar la cobertura y calidad de la planificación familiar, de ofrecer educación sexual y de establecer servicios para adolescentes, entre otras medidas destinadas a disminuir los embarazos no deseados, son menores que el costo de la atención de las complicaciones inmediatas y a largo plazo del aborto, más las repercusiones financieras que para la familia y la sociedad implican las muertes maternas. En nuestros países, donde la proporción del producto interno bruto dedicado a la atención de la salud está aún muy por debajo de las recomendaciones internacionales, resulta particularmente grave que estos recursos limitados se dedi- 
quen a problemas que pueden ser prevenidos con medidas sencillas y de bajo costo.

El aborto inducido es frecuente. El aborto inducido es, sin lugar a dudas, frecuente en ALC. Sin embargo, dada la naturaleza ilegal del mismo, no se cuenta con datos confiables sobre su incidencia. De hecho, los números varían en un intervalo muy amplio en función de la postura que frente al aborto inducido sustenta quien genera y difunde la información correspondiente.

Las únicas estadísticas nacionales con las que se cuenta se refieren al número de hospitalizaciones por aborto. Ciertas técnicas estadísticas permiten estimar, a partir de este dato, la frecuencia del aborto inducido. Según los resultados de un ejercicio llevado a cabo por el Instituto Alan Guttmacher, en seis países que reúnen al $70 \%$ de la población de América Latina (Brasil, Chile, Colombia, México, Perú y la República Dominicana) se realizan por año aproximadamente 2,8 millones de abortos inducidos (1). Si estos resultados se extrapolaran a toda la Región, el número de abortos inducidos por año alcanzaría unos 4 millones. Un fenómeno de esta magnitud, con las graves repercusiones ya mencionadas, constituye, sin lugar a dudas, un grave problema de salud pública.

El nivel socioeconómico de la mujer determina sus riesgos ante un aborto inducido. Los riesgos que una mujer enfrenta ante un aborto inducido son un reflejo de la desigualdad social que prevalece en ALC. Así, las mujeres con recursos que viven en áreas urbanas tienen la opción de interrumpir un embarazo no deseado en condiciones mucho más seguras que las que habitan en zonas rurales y son de bajo nivel socioeconómico. Las primeras suelen tener acceso a profesionales de la salud capacitados para hacer un aborto, mientras que las segundas suelen provocárselo ellas mismas o con la asistencia de una persona sin entrenamiento ni la infraestructura necesaria. Los métodos que las mujeres llegan a utilizar en el intento por resolver su problema solo pueden ser vistos como un reflejo de su desesperación: introducción de sondas o alambres, caídas por las escaleras, golpes que se dan ellas mismas o sus parejas. Por su parte, las mujeres con información y recursos acuden a profesionales que utilizan técnicas seguras, mitigan el dolor con anestésicos o analgésicos y suelen indicar antibióticos profilácticos para evitar infecciones (1). La inequidad es brutal, tal vez la más sobresaliente en el campo de la salud reproductiva. Baste recordar que un aborto inducido en condiciones óptimas repre- senta para la mujer menor riesgo que una inyección de penicilina o un parto normal.

En ALC, la mayoría de las constituciones nacionales establecen el derecho universal a la protección de la salud (24). Es, por lo tanto, una responsabilidad social ineludible responder a las necesidades de todas las mujeres por igual, sin distinción de clase social. Una sociedad que admita que algunas de sus mujeres accedan a un aborto inducido sin riesgos ni dificultades mientras que otras experimentan los peligros arriba mencionados, no es una sociedad democrática.

La calidad de la atención tras el aborto adolece de importantes limitaciones. Es común que las mujeres que padecen complicaciones derivadas de un aborto inducido acudan a servicios públicos en busca de un tratamiento para sus problemas. Desafortunadamente, la atención tras un aborto suele ser de baja calidad técnica y humana. En primer lugar, en la mayoría de los servicios se utiliza el legrado instrumental para evacuar la cavidad uterina, método peligroso en manos no entrenadas. Esta técnica sigue siendo la más común a pesar de que actualmente se cuenta con la aspiración endouterina, que es más sencilla, segura y barata. En segundo lugar, es importante recordar que las mujeres que interrumpen un embarazo no deseado están en alto riesgo de repetir la experiencia; no obstante, solo una muy baja proporción de las que acuden a un servicio para la atención de un aborto incompleto o de sus complicaciones son dadas de alta con la información necesaria o con un método anticonceptivo que las proteja de un nuevo embarazo no deseado. Finalmente, el trato que reciben las mujeres en estas circunstancias se caracteriza con demasiada frecuencia por la rudeza, la crítica abierta o solapada, las largas esperas y la falta de respeto a su pudor (25).

Son varias las circunstancias que contribuyen a esta situación: a la falta de recursos que aqueja a nuestros servicios de salud se agrega una actitud punitiva del personal o, por lo menos, de indiferencia ante las difíciles circunstancias físicas y emocionales que padecen las mujeres en estas circunstancias. En todo país, independientemente de la postura oficial ante el aborto inducido, es imprescindible reconocer que las complicaciones derivadas de este son un problema de salud pública de la más alta prioridad y que todos los esfuerzos por lograr una mejoría de la calidad de la atención están plenamente justificados.

Otras consecuencias del embarazo no deseado sobre la salud se derivan de que es más frecuente en los extremos de la edad reproductiva: 
durante la adolescencia y en la edad madura. En ambos períodos, los riesgos durante el embarazo, parto y puerperio son mayores que entre los $20 \mathrm{y}$ los 40 años, según se describe en la próxima sección.

\section{Embarazo en adolescentes}

El embarazo en mujeres de menos de 19 años es común en la región. Encuestas recientes señalan que el 14\% de las gestaciones en Bolivia en 1998 fueron de adolescentes (2), el 19\% en Colombia en 2000 (3), el 27\% en Nicaragua en 1998 (8) y el 17\% en Paraguay en 1990 (26). Otra manera de apreciar la magnitud del problema es calcular la proporción de menores de 19 años que están embarazadas o ya han tenido por lo menos un hijo cuando se realiza una encuesta. Esta categoría (adolescentes embarazadas o con un hijo o más) representó un $44 \%$ en Bolivia en 1994 (6), un 39\% en Colombia en 1995 (9) y un $41 \%$ en Paraguay en 1996 (27). En México, el $15,2 \%$ de las menores de 20 años ya tuvo su primer hijo (4). Esta alta frecuencia de embarazos en adolescentes se asocia, obviamente, con un inicio temprano de la vida sexual. En México, por ejemplo, el $11 \%$ de las adolescentes de la cohorte 1965-1969 inició su vida sexual activa antes de los 16 años. Esta fracción era de casi el doble entre las jóvenes de áreas rurales y cinco veces mayor en las mujeres sin instrucción, en comparación con las que concluyeron la primaria. Finalmente, otro dato interesante para ilustrar el problema del embarazo en adolescentes es que las parejas jóvenes utilizan anticonceptivos con menor frecuencia que los adultos para retrasar el primer embarazo (en México, solo el $17,5 \%$ de las parejas sin hijos lo hace). Esta baja utilización de anticonceptivos determina un alto riesgo de embarazo en las jóvenes (4).

En ALC no sabemos cuántos de los embarazos en adolescentes son deseados. Sin embargo, es de suponer que una buena proporción de estas madres jóvenes hubieran preferido evitarlos, de haber contado con los recursos para ello. La prevención de un embarazo no deseado en este grupo de edad es aún más trascendente que en otros momentos de la vida, debido a las consecuencias biológicas y sociales, tanto para la madre como para el niño, de los embarazos a edades tempranas:

- Las madres muy jóvenes (especialmente las menores de 15 años) tienen una mortalidad materna 2,5 veces mayor que las de 20 a 24 años (28-30).

- Las adolescentes corren mayor riesgo de ganar poco peso durante el embarazo, sufrir hipertensión inducida por el embarazo, anemia, infecciones de transmisión sexual y desproporción cefalopélvica (29).
- Las adolescentes corren mayor riesgo de sufrir violencia y abuso sexual.

- Ante un embarazo que la adolescente no está en condiciones de llevar a término, la joven suele carecer de conocimientos, recursos y orientación para resolver su situación. Sola y vulnerable, recurre a los métodos más peligrosos con el fin de interrumpir el embarazo. Asimismo, el miedo a la censura la lleva a retrasar la asistencia a los servicios de salud para buscar ayuda frente a las complicaciones derivadas de un aborto incompleto. Esta cadena de acontecimientos coloca a la joven en alto riesgo de enfermedad y muerte.

- Los hijos de madres menores de 15 años corren un riesgo dos veces mayor de tener bajo peso al nacer $(<2500 \mathrm{~g})$ y tres veces mayor de morir en los primeros 28 días de vida que los niños de madres de mayor edad (30).

- La incidencia de muerte súbita es mayor entre los hijos de adolescentes y, más adelante, estos niños también sufren con mayor frecuencia enfermedades y accidentes (28).

- Los hijos de madres adolescentes corren mayor riesgo de morir durante sus primeros 5 años de vida. Un estudio comparativo para el que se usaron datos de encuestas de demografía y salud de 20 países muestra que este riesgo es un $28 \%$ mayor para los hijos de adolescentes que para los de madres de 20-29 años (31). Esta mayor mortalidad es consecuencia de las condiciones en las que se crían estos niños, caracterizadas por la pobreza, los cuidados deficientes y una utilización menor y más tardía de los servicios de salud.

En general, este conjunto de problemas resulta de una combinación de factores: el bajo nivel socioeconómico, la inmadurez física de la madre, la falta de atención prenatal, los hábitos inadecuados relacionados con la salud (nutrición y tabaquismo, por ejemplo) y la inmadurez emocional.

El embarazo también tiene consecuencias sobre las oportunidades de desarrollo de las jóvenes. Así, se ha observado repetidamente que las adolescentes embarazadas abandonan la escuela con mayor frecuencia que el resto, tienen mayor probabilidad de ser madres solteras o solas, van a tener un número mayor de hijos al final de su vida reproductiva y sus oportunidades de alcanzar un trabajo remunerado son menores (12).

¿Cuántos embarazos no deseados y sus consecuencias se evitarían si las jóvenes y sus parejas recibieran una adecuada y oportuna educación sexual y tuvieran acceso a programas y servicios de planificación familiar? Aún cuando no se tiene una respuesta concluyente, es posible suponer que muchos. Sin lugar a dudas, las adolescentes y las parejas jóvenes deben ser un objetivo prioritario de 
todos los esfuerzos por prevenir embarazos no deseados, ya que estos se concentran en este grupo de edad y sus consecuencias son aun más graves que en otras etapas de la vida.

\section{Embarazo en mujeres mayores}

Hacia el final de la vida reproductiva las mujeres generalmente ya han tenido los hijos que deseaban o aun más de los que habían previsto en su juventud. Sin embargo, todavía son fértiles y pueden quedar embarazadas sin desearlo. Si una mujer de esta edad decide llevar adelante su gestación, los riesgos que enfrenta son mayores que en etapas más tempranas de la vida. Así, por ejemplo, la mortalidad y morbilidad maternas en madres mayores de 40 años son varias veces mayores que entre las más jóvenes $(32,33)$. Además, en esta etapa de la vida los problemas crónicos, tales como la diabetes y la hipertensión, son más comunes y pueden agravarse como resultado del embarazo. Los riesgos para el feto (defectos cromosómicos como el síndrome de Down, malformaciones congénitas, sufrimiento fetal y bajo peso al nacer) también son mayores que cuando la embarazada es más joven.

Esta mayor vulnerabilidad añade dramatismo a las alternativas que una mujer en esta etapa de la vida debe considerar ante un embarazo no deseado: la interrupción de la gestación y sus consecuencias, o su continuación, con los riesgos que conlleva. Ello sin mencionar los problemas que representa agregar un hijo más a una familia que a esas alturas probablemente ya sea numerosa.

\section{Suicidios y homicidios}

Ante un embarazo no deseado, una mujer puede recurrir al suicidio o ser víctima de un homicidio. Que una mujer acabe con su propia vida como respuesta a un embarazo no deseado es, seguramente, una de las consecuencias más trágicas de este problema. De igual forma, es inadmisible que la mujer pague con su vida el desacuerdo de su pareja o de otros miembros de la familia con el embarazo.

Diversos autores han expresado su preocupación sobre estas consecuencias del embarazo no deseado y se han referido a ellas como "el tema ausente" en los estudios sobre la mortalidad materna (34-36). En la mayoría de los países desarrollados ya prácticamente no existen el suicidio y el homicidio relacionados con un embarazo no deseado (34), probablemente porque en estos lugares las legislaciones y los sistemas de salud ofrecen mejores opciones y más protección a las mujeres que enfrentan este problema. Unos pocos estudios en países en desarrollo y, en particular, en América Latina, permiten apreciar la magnitud de este problema. En Matlab, Bangladesh, en el período 1976-1986 se demostró que de 409 muertes maternas, 17 (4\%) fueron suicidios y 8 (2\%) homicidios (37). En una región de Zimbabwe, en el período 1989-1990, se demostró que de 175 muertes maternas, 4 mujeres se suicidaron porque no deseaban el embarazo, cifra correspondiente al $2 \%$ de las muertes maternas en esa región (38). En un estudio realizado en una provincia de Argentina se registraron seis casos de embarazadas que fallecieron de muerte violenta entre 1992 y 1996: dos se suicidaron, una fue asesinada y tres murieron en accidentes (35). Algunos autores sugieren que este podría ser un problema creciente en países en desarrollo (34-36). La difícil situación económica y el temor a la crítica familiar y social, combinados con la ausencia de leyes y de un sistema social que proteja a las mujeres con embarazos no deseados, contribuyen seguramente a la elección del suicidio e incrementan la probabilidad de que las mujeres sean víctimas de la violencia de quienes se oponen a dicho embarazo. Esta es un área poco explorada que merece ser investigada en profundidad en la Región. En el estudio de autopsias verbales que se está llevando a cabo en México se van a analizar los casos de muerte violenta de mujeres en edad fértil, con la esperanza de documentar la proporción de mujeres embarazadas que fallecieron en tales circunstancias.

\section{Consecuencias del embarazo no deseado sobre la sociedad}

Impacto demográfico. La reproducción ha sido reconocida como un asunto de derechos humanos y de salud, y sus efectos sobre el crecimiento poblacional ya no se enfatizan, ni tampoco se utilizan metas demográficas como guía para políticas y programas (39). Sin embargo, es indudable que la fecundidad $^{4}$ afecta el perfil demográfico de una población. De hecho, los tres determinantes más importantes del crecimiento poblacional observado en el último siglo ${ }^{5}$ han sido la mortalidad, que disminuyó drásticamente como consecuencia de las mejores condiciones sanitarias, las vacunas, los antibióticos y otras intervenciones altamente eficaces; la

\footnotetext{
4 Número de hijos que las mujeres tienen durante su período reproductivo.

5 La población mundial ha aumentado de 1600 millones en 1900 a 2500 millones en 1950 y a 6000 millones en 2000 (40). Si no cambian los factores que contribuyen al crecimiento poblacional, se estima que en el mundo habrá 10400 millones de habitantes hacia el año 2100, y este incremento se deberá casi totalmente al aumento de la población en las regiones en desarrollo (41).
} 
fecundidad, que se mantuvo alta mientras la mortalidad disminuía, y la alta proporción de jóvenes en la estructura demográfica, que determina un elevado número de hijos en la siguiente generación, incluso cuando la fecundidad individual de cada uno de estos jóvenes es relativamente baja. ${ }^{6}$

En la mayoría de los países de la Región existe preocupación por el ritmo acelerado de crecimiento de la población. Sin embargo, existen excepciones, como Argentina, Chile y Uruguay, cuyas pirámides poblacionales corresponden más a las de países que han completado la transición demográfica. Un análisis detallado de estas diferencias excede los objetivos de este trabajo. Lo que sigue es relevante, sobre todo, para los países con un crecimiento poblacional todavía acelerado.

La fecundidad de una población resulta de las expectativas reproductivas (los hijos que las mujeres y las parejas desean tener) y de su acceso a los medios que les permiten cumplir con estas expectativas (especialmente los métodos anticonceptivos). Para que la fecundidad y, en consecuencia, el crecimiento demográfico de un grupo social, un país o una región disminuyan, es esencial que su población aspire a un menor número de hijos y tenga acceso a los medios para lograrlo. De esta manera, los embarazos no deseados se reducirían al mínimo.

La fecundidad ya no contribuye al aumento poblacional cuando se acerca a los llamados "valores de reemplazo". Esto sucede cuando cada pareja tiene solamente dos hijos, que sustituyen, demográficamente hablando, a sus padres en la siguiente generación. La fecundidad media en ALC fue de 2,7 en el año 2000 (41), es decir, mayor que la fecundidad de reemplazo. ${ }^{7}$ Este valor medio abarca países y grupos con una fecundidad mucho más alta aún, que crecen a ritmos acelerados. Según los datos que arrojan las encuestas, el número de hijos al que las mujeres aspiran es menor que el que realmente tienen. La diferencia representa a los embarazos no deseados. Por ello, para lograr un crecimiento poblacional menor en la región es esencial, en primer lugar, que las personas logren concretar sus expectativas reproductivas y evitar los embarazos no deseados y, en segundo término, que deseen un número aún menor de hijos que los que quieren actualmente.

Si las expectativas reproductivas se cumplieran, las mujeres, parejas y familias no enfrentarían las situaciones y decisiones difíciles que se asocian con embarazos o hijos no deseados; además, la so-

\footnotetext{
6 El cuarto factor es la migración, que no se abordará en este documento porque su efecto es menos importante y porque no existe una relación clara con el tema del embarazo no deseado.

7 La fecundidad en ALC es mucho menor que en otras regiones en desarrollo: se estima una fecundidad de 5,1 en Africa y de 3,4 en el sur de Asia.
}

ciedad en general se beneficiaría, ya que el crecimiento poblacional y las demandas que se derivan de él serían menores. El impacto demográfico del descenso de la fecundidad a valores más cercanos a los de reemplazo no sería despreciable: para el año 2100 se estima que, si la fecundidad continúa como está actualmente, habrá una población de 8900 millones en las regiones subdesarrolladas y que la cifra sería de 7800 millones si la fecundidad disminuyera a 2 (42).

Se ha comprobado que el inicio tardío de la reproducción y el deseo de menos hijos se asocian con un mayor desarrollo social y, en especial, con el nivel de escolaridad de la madre. Por ello, los esfuerzos por influir en las expectativas reproductivas deben concentrarse en los jóvenes (hombres y mujeres), ya que ellos representan el mayor grupo en la estructura demográfica actual, son los que van a reproducirse en las próximas décadas y, sobre todo, son los más abiertos a cambiar la visión tradicional del papel de la mujer en la sociedad. Cuantas más oportunidades e incentivos existan para que las mujeres reciban educación y se desarrollen como trabajadoras o profesionales, más se retrasará la edad al nacer el primer hijo y más bajo será el número de descendientes que la mujer y su pareja deseen. $\mathrm{Si}$, al mismo tiempo, se garantizara el acceso efectivo a la anticoncepción para toda la población, el crecimiento de la población sería menor.

Hijos no deseados y criminalidad. Diversos investigadores han intentado identificar las circunstancias de la vida o los factores que contribuyen a la delincuencia juvenil. Dos importantes estudios han demostrado que el rechazo materno, el trato rudo o errático y la falta de supervisión por parte de los padres son factores que contribuyen a que los hijos participen en actividades delictivas durante la juventud $(43,44)$. En el mismo sentido, autores finlandeses encontraron que, en orden decreciente, el bajo nivel educativo de la madre, el hecho de ser madre adolescente, la crianza por solo uno de los padres, el que la madre no haya deseado el embarazo y el tabaquismo durante el mismo son factores que incrementan el riesgo de que el hijo sea un delincuente juvenil (45). Estas y otras investigaciones ofrecen pruebas sólidas para sustentar que los hijos que no fueron deseados y que crecieron en un ambiente desfavorable (bajo el cuidado de madres inexpertas, solas, con problemas que probablemente les impedían dar una atención adecuada a sus hijos) son más susceptibles de involucrarse en actividades criminales durante la juventud. Otros problemas sociales gravísimos, como la violencia doméstica y los niños de la calle, también están fuertemente asociados con la maternidad no dese- 
ada.

Recientemente, una investigación realizada por dos prestigiosos economistas de los EE. UU. encontró una asociación entre la legalización del aborto y el descenso de la criminalidad 15 a 20 años después de aprobada la ley correspondiente en ese país (46). Sus cálculos les permiten afirmar que la legalización del aborto es la primera causa del importante descenso de la tasa de asesinatos, daños a la propiedad y crimen violento en general que se ha observado en ese país durante la última década. De hecho, el $50 \%$ del descenso de la delincuencia podría atribuirse a la legalización del aborto.

En un esfuerzo por comprender esta asociación, los autores sugieren que tal vez las mujeres recurran al aborto como una manera de elegir el mejor momento de su vida para tener hijos. Ellos sostienen que la capacidad de una madre para ofrecer un ambiente favorable para el crecimiento de un niño varía según su edad, educación, ingresos, presencia del padre, el deseo de quedar embarazar y el consumo de alcohol, tabaco y otras drogas durante el embarazo. En consecuencia, el aborto legal le da a la mujer la oportunidad de retrasar el nacimiento de un hijo hasta que las condiciones sean favorables.

Conscientes de la controversia que sus hallazgos despertarían, los autores se ocupan de aclarar enfáticamente que ellos no tienen una posición especialmente favorable ante el aborto ni están proponiéndolo como una solución a ningún problema social. De hecho, dicen que efectos similares se podrían lograr con otras medidas, como una anticoncepción más eficaz o programas que aseguren el crecimiento en un ambiente favorable para todos los niños con riesgo de involucrarse en actividades criminales más adelante en sus vidas.

\section{CONCLUSIONES}

En este documento se ofrece información y se discuten argumentos que permiten afirmar que el embarazo no deseado es un problema común en la Región, con consecuencias graves sobre la salud y la situación social de las mujeres, sus hijos y familias, así como sobre la población en general. Además, también se ha dejado claro que el embarazo no deseado no es solo una responsabilidad individual y de la pareja, sino de toda la sociedad. A continuación se describen las medidas más importantes que deberían promoverse para reducir este problema y su impacto.

\section{Prevención del embarazo no deseado}

- En países en los que las mujeres acceden a niveles más altos de educación formal y su inserción en el mercado laboral es creciente, las expectativas reproductivas cambian y ellas retrasan la edad de unión, el inicio de la vida sexual y el nacimiento del primer hijo, al mismo tiempo que aspiran a formar una familia más pequeña. Para que la mujer no sufra las consecuencias de la contradicción entre lo que quiere y necesita y lo que se espera de ella, es fundamental amortiguar las presiones de la pareja, la familia y la sociedad en favor de una unión y maternidad tempranas y una progenie numerosa. Para lograrlo, es necesario que se establezcan programas amplios de comunicación social en los que se resalten el derecho de las mujeres a un desarrollo personal pleno y los beneficios que para ellas mismas, la familia y la sociedad resultan de su mejor educación e inserción en el trabajo. Al mismo tiempo, es esencial introducir en los sectores educativo, de salud y laboral una perspectiva de género que asegure que las mujeres reciban un trato justo, tengan oportunidades especiales que les permitan avanzar hacia una situación equitativa y cuenten con programas y servicios que hagan compatibles la vida familiar con las exigencias de los estudios o el trabajo.

- Para que las expectativas reproductivas puedan concretarse es fundamental poner al alcance de todos, pero en especial de los jóvenes, programas y servicios de educación sexual y reproductiva, condición fundamental para que los adolescentes se hagan responsables de su sexualidad y no enfrenten riesgos derivados de esta. Sin acceso a información oportuna y de buena calidad, los jóvenes motivados no podrán concretar sus aspiraciones. Esta información deberá ser veraz y objetiva, sin prejuicios ni barreras morales. Los sectores de la educación y la salud tienen la responsabilidad fundamental de ofrecerla en todas las oportunidades, adaptando los mensajes a las características culturales de aquellos a quienes van dirigidos.

- Además de la información sobre sexualidad y reproducción, la prevención de embarazos no deseados solo podrá lograrse si los gobiernos y la sociedad civil ofrecen programas y servicios de anticoncepción accesibles y de buena calidad. Esta es, obviamente, condición esencial para prevenir los embarazos no deseados. Para aumentar la cobertura se deben establecer servicios para la población más difícil de alcanzar, con necesidades especiales: los jóvenes, los hombres, las mujeres de grupos étnicos y comunidades rurales dispersas.

Para mejorar la calidad de los servicios, es esencial dar más y mejor información, adaptada a la 
cultura y nivel educativo del paciente, asegurar que el trato humano sea cálido y respetuoso, y reforzar la capacidad técnica de los profesionales de la salud que ofrecen los métodos. Asimismo, debe ampliarse la gama de anticonceptivos disponibles para responder a la diversidad de necesidades y preferencias de los potenciales usuarios. Finalmente, también es recomendable que se aproveche todo contacto con las personas que acuden a los servicios de salud para promover la anticoncepción y ofrecer servicios. Es importante recordar que el mal uso o la falta de uso de anticonceptivos es muchas veces el resultado de la pobreza de la información que se proporciona a los pacientes, de la falta de discusión sobre sus necesidades y de las limitadas opciones anticonceptivas disponibles.

Además de los métodos que regularmente se ofrecen en la región (esterilización, anticonceptivos orales, dispositivo intrauterino, condón e inyectables), en los últimos años se han hecho enormes esfuerzos por informar y poner la anticoncepción de emergencia al alcance de todos, pero especialmente de las víctimas de la violencia sexual y de los jóvenes. Si la cobertura de planificación familiar fuera más amplia, la información y comunicación entre prestadores y usuarios de mejor calidad y la gama de métodos disponibles más amplia, el número de embarazos no deseados sería sustancialmente menor.

\section{Atención a las consecuencias del embarazo no deseado}

Cuando una mujer queda embarazada sin desearlo, sus alternativas son básicamente dos: intentar interrumpir el embarazo mediante un aborto inducido, o continuar con el mismo y dar a luz a un hijo que, probablemente, tampoco será deseado. Es un compromiso de todos responder ante ambas situaciones, ya que los embarazos no deseados son, en gran medida, una responsabilidad social.

Para mitigar las consecuencias del aborto inducido es esencial:

- Revisar la legislación vigente sobre interrupción voluntaria del embarazo y asegurar que, cuando se cumplan las causales para acceder a un aborto legal, las mujeres no encuentren barreras para recibir el servicio ni sufran un trato discriminatorio y de calidad deficiente. Es intolerable observar como ni siquiera las niñas violadas por sus padres o las mujeres con retraso mental que sufren abuso sexual tienen acceso a un aborto legal en muchos países de nuestra Región, aunque los instrumentos legales establezcan claramente su derecho al aborto en estas circunstancias.
Las causales que permiten un aborto legal varían en los distintos países de la región (24). Los extremos están representados por Chile y El Salvador, donde el aborto está prohibido en toda circunstancia, y Cuba y Guyana, países que lo autorizan sin ninguna restricción. Entre estos dos extremos, la legislación varía, aunque en la mayoría de los países el aborto está permitido cuando el embarazo fue resultado de una violación o cuando está en peligro la vida de la madre. Existen algunos intentos recientes en lugares específicos (como la ciudad de México) para ampliar la legislación y agregar otras circunstancias como el peligro para la salud de la mujer embarazada.

- Garantizar todas las mujeres el acceso a servicios para la atención del aborto incompleto o complicado, según se establece en el programa de acción de El Cairo y en la mayoría de las constituciones de la Región (derecho a la protección de la salud) $(24,39)$. La atención tras el aborto debe incluir la evacuación uterina completa con técnicas eficaces y seguras, la atención a las complicaciones y asesoramiento y servicios de planificación familiar de calidad. Es lamentable que los servicios de salud no cuenten con personal adiestrado para la interrupción legal del embarazo. De hecho, en la actualidad existen métodos instrumentales y farmacológicos que son muy seguros y eficaces. Los sistemas de salud deberían procurar poner estas nuevas tecnologías al alcance de quienes las necesitan.

Los buenos servicios de atención a las complicaciones del aborto contribuyen a evitar embarazos no deseados, ya que las mujeres que han tenido un aborto inducido corren mayor riesgo de tener otro subsecuente, como resultado de la persistencia de las mismas circunstancias que contribuyeron al primero (falta de información o de acceso a servicios de anticoncepción, entre otros). Si durante la atención tras el aborto se establece una buena relación con la paciente, se le da información sobre la reproducción y la anticoncepción y se le ofrecen métodos adecuados, se habrá contribuido a romper este ciclo y se ayudará a miles de mujeres.

Tan esencial como ayudar a las mujeres que recurren al aborto para evitar un hijo no deseado, es apoyar a aquellas que continúan con el embarazo mediante:

- Programas de apoyo especial para madres solteras y jefas de hogar, para ayudarles a hacer compatibles sus tareas fuera y dentro del hogar.

- Revisión de la legislación laboral vigente para eliminar la importante discriminación que aún sufren las mujeres, caracterizada por la "doble 
jornada" (trabajo remunerado fuera del hogar, sumado a las actividades domésticas), la jefatura de hogar femenina y los menores salarios a igualdad de responsabilidades en comparación con los hombres.

- Oportunidades especiales para que las mujeres adquieran autonomía económica, como subsidios y préstamos para el establecimiento de microempresas.

- Apoyo a las adolescentes para que emprendan proyectos productivos.

- Becas de estudios para contribuir al desarrollo de las jóvenes y al cambio consecuente en sus expectativas reproductivas.

- Campañas para evitar la discriminación de las adolescentes embarazadas en las escuelas, situación que las lleva a abandonar sus estudios.

- Revisión de la legislación y de los procedimientos para la adopción, con el fin de facilitarla.

- Promoción del compromiso del hombre en la práctica anticonceptiva y en la salud reproductiva, en general.

- Esfuerzos multisectoriales constantes para avanzar hacia una mayor igualdad entre los géneros en todos los ámbitos.

Los programas oficiales, la opinión mundial y los mensajes que reciben las parejas por distintos canales promueven una familia poco numerosa. Sin embargo, los mismos que sustentan esta postura no parecen reconocer la responsabilidad social ante el embarazo no deseado. Mientras la mujer sufre las graves consecuencias del aborto clandestino sobre su salud, seguridad y dignidad, la sociedad cierra los ojos ante el hecho de que los programas de anticoncepción tienen una cobertura y calidad deficientes, y de que los limitados recursos para la atención de la salud se están gastando en tratar, con una calidad limitada por cierto, las complicaciones de abortos que podrían evitarse. Si los abortos y sus complicaciones se previnieran, los recursos podrían orientarse hacia otros problemas de salud reproductiva, para los que las soluciones no están al alcance todavía. Además de estas responsabilidades que la sociedad no debería soslayar, es importante agregar una más: millones de embarazos no deseados culminan en el nacimiento de niños cuyas necesidades básicas no pueden ser satisfechas por sus madres y familias. Enfrentar y encontrar soluciones para los niños y jóvenes abandonados, que viven en condiciones de carencia física, emocional y social, es también una responsabilidad ante la que ningún miembro responsable de la sociedad puede cerrar los ojos.

\section{SYNOPSIS}

\section{Unwanted pregnancy: impact on health and society in Latin America and the Caribbean}

Engaging in sexuality and reproduction should always be something that is wanted and planned. Unfortunately, when that is not the case, one result can be unwanted pregnancy. Unwanted pregnancies have consequences for women, their families, and their countries.

This document reviews the causes and results of unwanted pregnancy, emphasizing the impact that this problem has on Latin America and the Caribbean (LAC). Four reasons why unwanted pregnancy is a continuing problem in LAC are: 1) people's growing desire to have smaller families, 2) the unmet need for family planning, 3) the fact that contraceptive methods are not $100 \%$ effective, and 4) unwanted sexual relations. Unwanted pregnancies especially affect adolescent women, single women, and women over 40 years of age. Given their desperate situation with an unwanted pregnancy, some women opt for an unsafe abortion, which can lead to their death. Other women can go so far as to commit suicide, or be murdered by a family member or other person who is unhappy that the pregnancy has occurred. It has been found that women who decide to continue with the pregnancy have higher risks of suffering an illness, and the same is true for the child.

Reducing unwanted pregnancies and treating postabortion complications are key to lowering maternal mortality and morbidity. This necessitates developing mass communication programs that address gender issues, education programs for girls, and sex education programs. It is also vital to make available to all persons reproductive health services that include family planning methods. In the countries of LAC with laws that specify grounds for legally ending a pregnancy, it is necessary that health care be organized to actually provide this service, and that health care programs obtain the safest, most effective technologies now available

\section{REFERENCIAS}

1. The Alan Guttmacher Institute. El aborto clandestino: una realidad latinoamericana. Nueva York: The Alan Guttmacher Institute; 1994

2. Demographic and Health Survey. Encuesta Nacional de Demografía y Salud, Bolivia. Calverton, MD: Macro International; 1998.
3. Demographic and Health Survey. Encuesta Nacional de Demografía y Salud, Colombia. Calverton, MD: Macro International; 2000.

4. Consejo Nacional de Población. Cuadernos de Salud Reproductiva. México, DF: Consejo Nacional de Población; 2000.
5. Demographic and Health Survey. Encuesta Nacional de Demografía y Salud, Bolivia. Calverton, MD: Macro International; 1989.

6. Demographic and Health Survey. Encuesta Nacional de Demografía y Salud, Bolivia. Calverton, MD: Macro International; 1994. 
7. World Fertility Survey. Encuesta sobre Salud Familiar. Nicaragua. Atlanta, GA: Centers for Disease Control; 1993.

8. Demographic and Health Survey. Encuesta Nacional de Demografía y Salud, Nicaragua. Calverton, MD: Macro International; 1998.

9. Demographic and Health Survey. Encuesta Nacional de Demografía y Salud, Colombia. Calverton, MD: Macro International; 1995.

10. Encuesta Nacional de la Dinámica Demográfica, ENADID. México, DF: Instituto Nacional de Estadística Geografía e Informática; 1997.

11. Langer A. Hacia una maternidad sin riesgos: acciones para México en el contexto internacional. En Elu M y Santos Pruneda E (eds.). Una nueva mirada a la mortalidad materna. México: Comité Promotor por una Maternidad sin Riesgos en México; 1999. Pp. 29-39.

12. Brown S, Eisenberg L (eds.). The best intentions: unintended pregnancy and the well-being of children and families/ Committee on Unintended Pregnancy. Washington, DC: Institute of Medicine, National Academy of Sciences; 1995.

13. Hardy E, Costa G. Abortion experience among female employees of a Brazilian university. Campinas, SP: CEMICAMP; 1993.

14. Hernández D, Mojarro $\mathrm{O}$, Fuentes J, Martínez-Manatou J. Consideraciones sobre las muertes maternas en el IMSS y sus causas. México, DF: Instituto Mexicano del Seguro Social; 1991.

15. Dagg P. The psychological sequelae of therapeutic abortion-denied and completed. Am J Psychiatry 1991;148:578-585.

16. Koonin L. Maternal mortality surveillance, United States. MMWR 1991;37 (supl 5):19-29.

17. Muñoz L, Ñañez H, Becerra E, Klevens J. Mortalidad materna en el Instituto Materno-Infantil de Bogotá (1976-1980). Revista de la Facultad de Medicina 1985; 39:331-351

18. Reyes S. Mortalidad materna en México. México, DF: Instituto Mexicano del Seguro Social; 1994.

19. Hernández B, Langer A, Romero M, Chirino J. Factores asociados a la muerte materna hospitalaria en el estado de Morelos, México. Salud Publica Mex 1994;36:521-528.

20. La Guardia K, Rothoz M, Belfort P. A 10year review of maternal mortality in a municipal hospital in Rio de Janeiro: a cause for concern. Obstet Gynecol 1990; 75:27-32.

21. World Health Organization, Division of Reproductive Health. Unsafe abortion. Global and regional estimates of incidence of and mortality due to unsafe abortion, with a listing of available country data. Geneva: WHO; 1998. (WHO/RHT/MSM/97).

22. Royston E. Estimating the number of abortion deaths. Methodological issues in abortion research. Nueva York: Population Council, IPAS, WHO; 1991.

23. Rajs D. Maternal Mortality. En Timaeus I, Chackiel J, Ruzicka L. (Eds) Adult Mortality in Latin America. Oxford: Clarendon Press; 1996. pp. 276-294.

24. The world's abortion laws, February 1999. [Sitio en Internet The Center for Reproductive Law and Policy. Disponible en: http://www.crlp.org/pub_fac aborlicpd.html. Acceso el 10 de agosto de 2001.

25. Langer A, García C, Heimburger A, Stein K, Winikoff B. Assessing the quality of post-abortion care in a public hospital of Oaxaca, Mexico. Reproductive Health Matters 1997;9:20-28.

26. Demographic and Health Survey. Encuesta Nacional de Demografia y Salud, Paraguay. Calverton, MD: Macro International; 1990.

27. World Fertility Survey. Encuesta Nacional de Demografía y Salud Reproductiva, Paraguay. Atlanta, GA: Centers for Disease Control; 1996.

28. Morris L, Warren C, Aral S. Measuring adolescent sexual behaviors and related health outcomes. Public Health Rep 1993;108(supl 1):31-36.

29. Stevens-Simon C, White M. Adolescent pregnancy. Pediatr Ann 1991;20:322-331.

30. McAnarney E, Hendee W. Adolescent pregnancy and its consequences. JAMA 1989;262:74-77.

31. Bicego G, Ahmad OB. Infant and Child Mortality, DHS Comparative Studies No. 20. Calverton, MD: Macro International; 1996.

32. Koonin L, Atrash H, Lawson H, Smith J. Maternal mortality surveillance, United States. MMWR 1991;40:1-13.

33. Prysak M, Lorenz R, Kisly A. Pregnancy outcome in nulliparus women 35 years and older. Obstet Gynecol 1995;85:65-70.

34. Frautschi S, Cerulli A, Maine D. Suicide during pregnancy and its neglect as a component of maternal mortality. Int J Gynaecol Obstet 1994:47:275-284.

35. Rizzi R, Cordoba R, Maguna J. Maternal mortality due to violence. Int J Gynaecol Obstet 1998;63(supl 1):19-24.

36. Ronsmans C, Khlat M. Adolescence and risk of violent death during pregnancy in Matlab, Bangladesh. Lancet 1999;354 (9188):1448.

37. Fauveau V, Blanchet $T$. Deaths from injuries and induced abortion among rural Bangladeshi women. Soc Sci Med 1989; 29:1121-1127.

38. Mbizvo M, Fawcus S, Nystrom L. Maternal mortality in rural and urban Zimbawe: social and reproductive factors in an incident case-referent study. Soc Sci Med 1993;36:1197-1205

39. Family Care International. Meeting the Cairo Challenge. A Summary Report, 1999. New York: Family Care International; 1999.

40. World Bank. World Development Indicators. New York: World Bank; 1998.

41. World Population Prospects: The 1998 Revision. Annex I: Demographic indicators. Nueva York: United Nations Department for Economic and Social Information and Policy Analysis, Population Division; 1998.

42. Bongaarts J, Bulatao R. Completing the demographic transition. New York: Population Council; 1999. Working Paper No. 125.

43. Loeber R, Stouthamer-Loeber M. Family factors as correlates and predictors of juvenile conduct problems and deliquency. En: Tonry M, Morris N. (Eds) Crime and justice, VII. Chicago: University of Chicago Press; 1986.

44. Sampson R, Laub J. Crime in the making: pathways and turning points through life. Cambridge, MA: Harvard University Press; 1993.

45. Rasanen $P$, Hakko $H$, Isohanni M, Hodgins S, Jarvelin M, Tiihonen J. Maternal smoking during pregnancy and risk of criminal behavior among adult male offspring in the Northern Finland 1966 Birth Cohort. Am J Psychiatry 1999;156: 857-862.

46. Donohue J, Levitt S. The impact of legalized abortion on crime. Cambridge, MA: National Bureau of Economic Research; 2000. Working Paper 8004. 
for ending a pregnancy. 